\title{
Agro Drone
}

\author{
Rajath.C ${ }^{1}$, Rushikesh.S.D ${ }^{2}$, Rishab.B ${ }^{3}$, Ruthvik.R ${ }^{4}$ \\ Alumni, Department of ECE, B.N.M Institute of Technology, Bangalore, India ${ }^{1-4}$
}

\begin{abstract}
Agriculture is the backbone of many developing nations and plays a major role in their economy. In our country, it accounts for $13.9 \%$ of Indian GDP and about $50 \%$ of the workforce. But the yield is poor because of backward traditional farming approach and techniques. If we adapt a new technology with olden farming practices, it will be possible to boost the overall yield and improve the condition of agriculture in our country. We decided to implement this project for agricultural survey by using a UAV to help farmers improve crop quality and increase their earnings. The agriculture farm is surveyed by an infrared camera which shows the difference between infected or diseased crops and matured crops, it can also show weeds or pests and to a certain extent monitor the soil moisture levels. The drone can be used spray pesticides or irrigate the crops. The innovative frame design will allow the drone to be transported safely and easily.
\end{abstract}

Keywords: UAV, APM, OpenCV, ESC, NDVI

\section{INTRODUCTION}

An Unmanned Aerial Vehicle (UAV) commonly called a drone, is an aircraft which has no human pilot aboard. They are a part of Unmanned Aircraft System (UAS), which consists of UAV, ground-based controller and a communications system. The flight of UAVs may have different degrees of autonomy. It can either be controlled by remote by a human operator or autonomously by on-board computers. A drone can be used for agriculture monitoring, where the UAV is used for proper and accurate surveying of crops. In the proposed system, there is an on-board IR camera which monitors the crops to check for pests and notifies the user immediately if pests or worms are detected. The user can then attach the pesticide containing vessel to the drone and use it to spray the pesticide across the field through the nozzles present on each leg of the drone frame.

\section{Proposed SySteM}

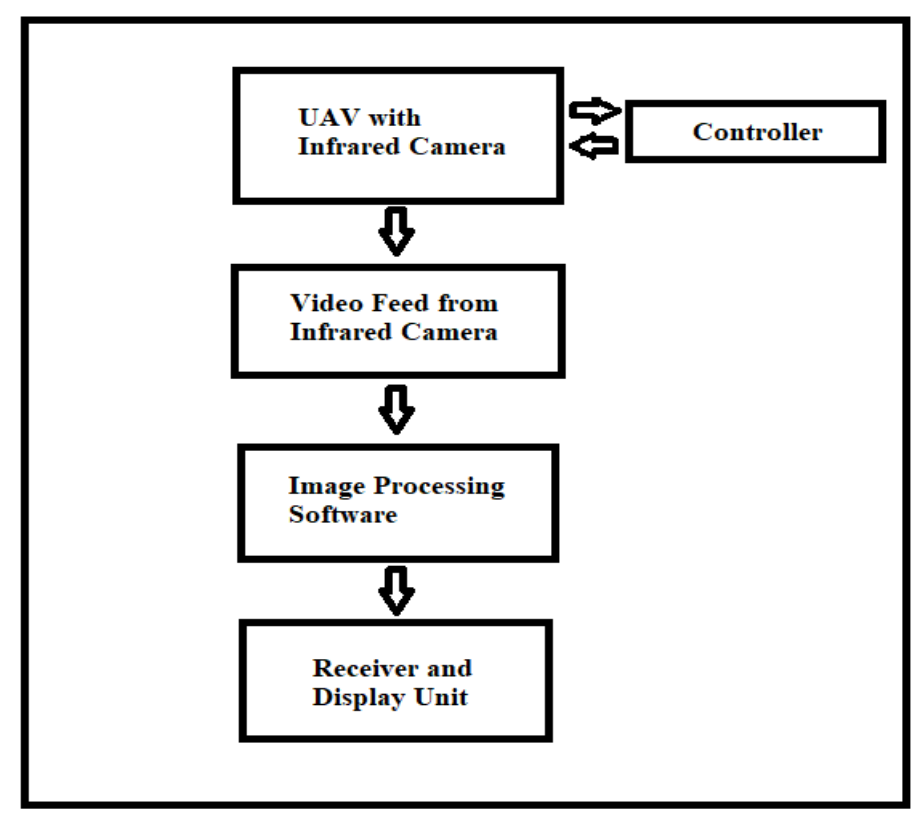

Figure 1. Block diagram of the proposed system

The block diagram is as shown in Figure 1. The infrared or IR camera monitors the designated crop area. The video feed is then analysed to determine if the crop is infected or withered. Once this situation is encountered, the user is immediately notified to take the needful action. The user can then spray pesticide or insecticide or simply remove the infected crop to prevent the other crops from being infected. The drone is controlled by means of remote control and 
therefore the farmers will not be directly in contact with the pesticides and insecticides which have adverse effects on their health. The pesticide container can be switched out for a water container and the same system can be used to irrigate the fields or for crop dusting purposes.

\section{COMPONENTS USED}

\section{MOTOR}

The motor used in the drone is a DC brushless motor which consists of a stack of laminated magnetic material and a set of miniature coils. The stator windings are excited by conventional DC brushless motor controllers. A direct current will be passed through three or more non-adjacent windings together, and the group of windings thus energized will be alternated electronically based upon rotor position feedback.

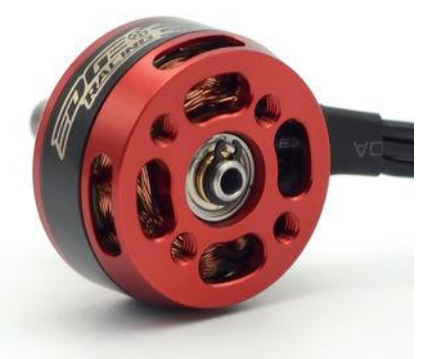

Figure 2. A typical out runner type DC Brushless Motor

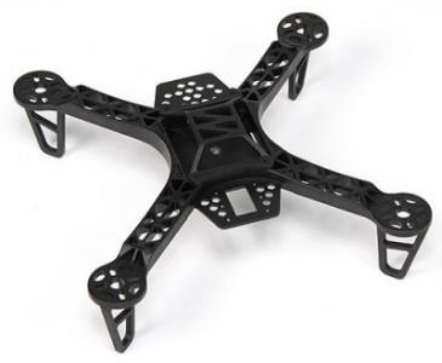

Figure 3. A Quad frame

\section{FRAME}

Frame used here is a carbon fibre Spyder frame of quad design which is extremely light weight and steady. It has a lifting capacity of up to 2 kilograms. The carbon fiber frame is used to cut costs and obtain a compact, robust and efficient design

\section{ELECTRONIC SPEED CONTROLLER}

ESC is an electronic circuit used to vary the motor's speed and possibly also to act as a dynamic brake. It allows much smoother and more precise variation and in a very efficient manner. The ESC used here is a 60A ESC which can give a boost current of up to 70A for 10 seconds.

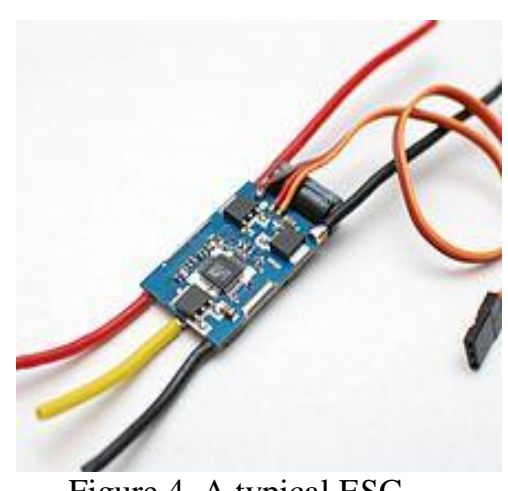

Figure 4. A typical ESC

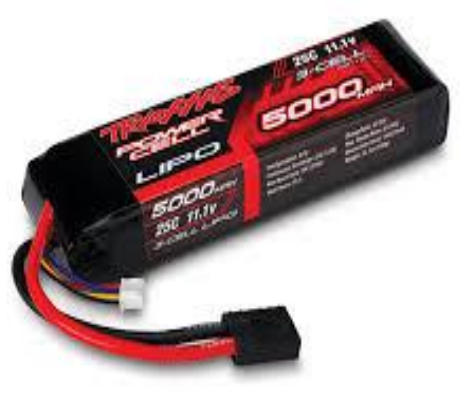

Figure 5.5000mAh Battery

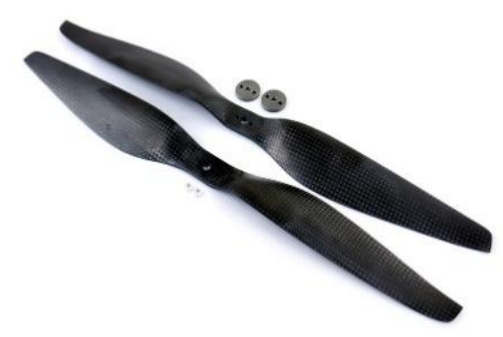

Figure 6. 10-inch Propellers

\section{PROPELLERS}

Propellers are used to convert raw rotational power into thrust which basically lifts the drone, moves it forward and also hover in mid-air. The propellers used here are 2 blade 12 inch carbon fibre blades which are extremely light and very strong.

\section{BATTERIES}

Batteries are the main driving energy of a drone. They come in different chemistries like Li-Po, Li-Ion, NiMH and many more. The battery used in this drone is a Li-Po Batteries for their high discharge rate per weight of the battery. These are used where weight is a critical factor like this drone. The battery used is a $5000 \mathrm{mAh}$ with $\mathrm{c}$ rating of $40 \mathrm{C}$. 


\section{APM CONTROLLER}

ArduPilotMega is professional Quality auto pilot that is based on Arduino Mega Platform. It is a full auto pilot capable of autonomous stabilization with point to point based navigation and telemetry capabilities via Zigbee. APM 2.8 was used in the system which has a built-in compass which is highly advantageous in drones and rovers. It allows the user to turn away fixed, rotary wing or multirotor vehicle into fully autonomous systems with advanced capabilities.

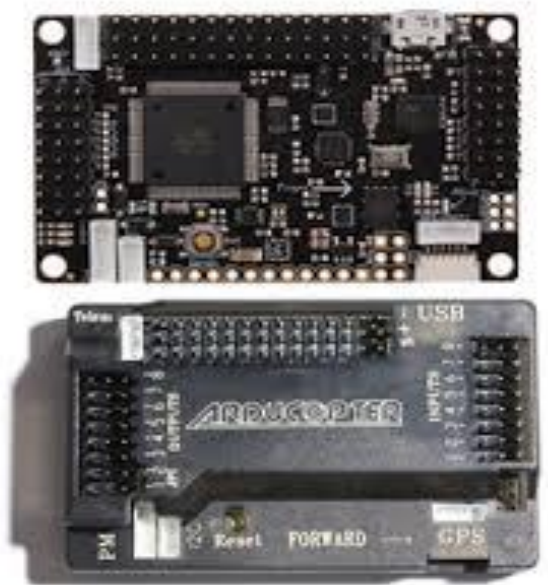

Figure 7. APM Controller

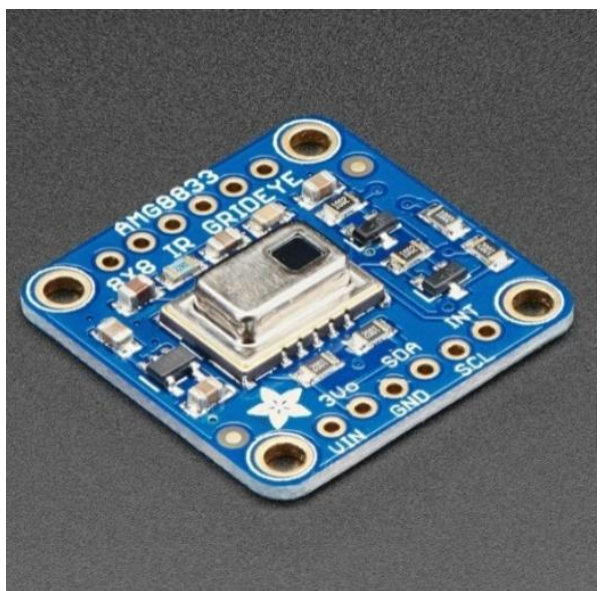

Figure 8. IR Camera

\section{INFRARED (IR) CAMERA}

An infrared camera is a device which detects infrared energy or heat and converts it into an electronic signal that is then processed to produce a thermal image which can be used for future study. An infrared camera with thermal imaging capability is used in the proposed system.

\section{RADIO CONTROLLER}

The radio controller used is a $2.4 \mathrm{GHz} 9$ channel controller. It has telemetry capabilities and can be used to effectively control the motion of the drone.

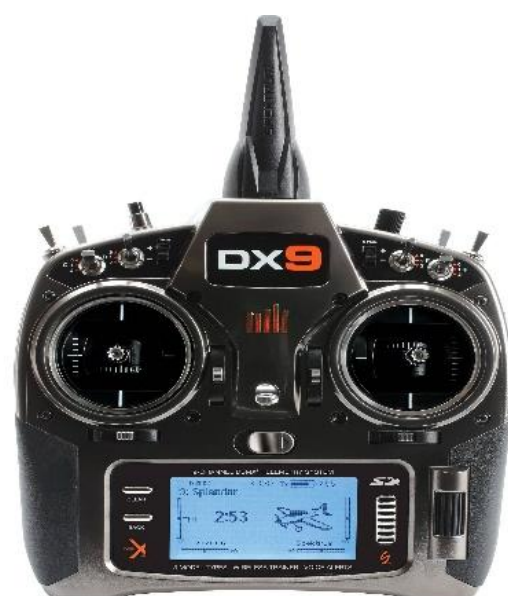

Figure 9. RC Controller

\section{Methodology}

We use a UAV fitted with an infrared camera for surveillance of the crops. The video feed received from the camera undergoes image processing and by applying required filters, we will be able to distinguish between healthy crops, defective crops and/or pests. The video can be seen on the receiver and display unit. The remote controller is used to move the drone as per requirement to position it over the field. 


\subsection{CROP MONITORING}

The old age methods of measuring vegetation are although reliable; however, they are labor intensive, time consuming and only viable for areas of limited size. The latest developments in remote sensing provides more accurate data for vegetation parameters than old age techniques because it gives a coverage over a large area at repetitive intervals. Vegetation spectral reflectance characteristics depend on completely on the leaves properties the amount of energy or light reflected for a certain wavelength depends on the technical characters of leaf pigmentation, thickness, cells composition, and on the amount of liquid or water content in the leaf tissue. Green leafy plants typically display comparatively low reflectance and transmittance in visible region of spectrum by contrasts in near infrared region reflectance increases by far greater levels since there is considerable scattering at mesophyll cell wall interfaces and due to minimal absorbance by pigments, which are the factors controlling this part is leaf structure. The difference in reflectance between the visible and NIR wavelength is used for most of monitoring crop area. During pathogen infection, the infected tissue changes, such as difference in chlorophyll levels or even cell death. Thermal infrared is a useful tool for the ability to detect and show pathogens.

UAVs observe the crop area with different angles, Thus UAVs can cover up hectares of fields in single flight. For this observation thermal and multi spectral Cameras are used to record reflectance of vegetation, which is mounted to downside of the quad copter. The camera captures at pictures at 1 capture per second and stores it into memory and sends to the ground station through telemetry. For this wireless communication it uses MAVLINK protocol. The pictures are captured in the visible five brands with different wave lengths: i.e. (i) Blue wavelength 440-510nm, (ii) Green wavelength 520-590nm, (iii) Red wavelength 630-685nm, (iv) Red edge wavelength 690-730nm, (v) Near infrared wavelength $760-850 \mathrm{~nm}$. The data coming from the multispectral camera through telemetry is analyzed by Normalized Difference Vegetation Index (NDVI) represented in equation. 1.

$\mathrm{NDVI}=\left(\mathbf{R}_{\mathrm{NIR}}-\mathbf{R}_{\mathrm{RED}}\right) /\left(\mathbf{R}_{\mathrm{NIR}}+\mathbf{R}_{\mathrm{RED}}\right)$

where, $\mathbf{R}_{\mathbf{N I R}}=$ Reflectance of the near infrared band, $\mathbf{R}_{\mathbf{R E D}}=$ Reflectance of the red band. The calculations give the values -1 to +1 ; near to 0 (ZERO) indicates no vegetation on the crop and near to $+1(0.8$ to 0.9$)$ means highest density of green leaves on the crop. Based upon these results, farmers will easily identify the field where can spray the pesticides. The inbuilt GPS module maintains GPS coordinates of every captured picture. Then the GPS coordinates of that pictures are stored in UAV to spray pesticides automatically without manual control.

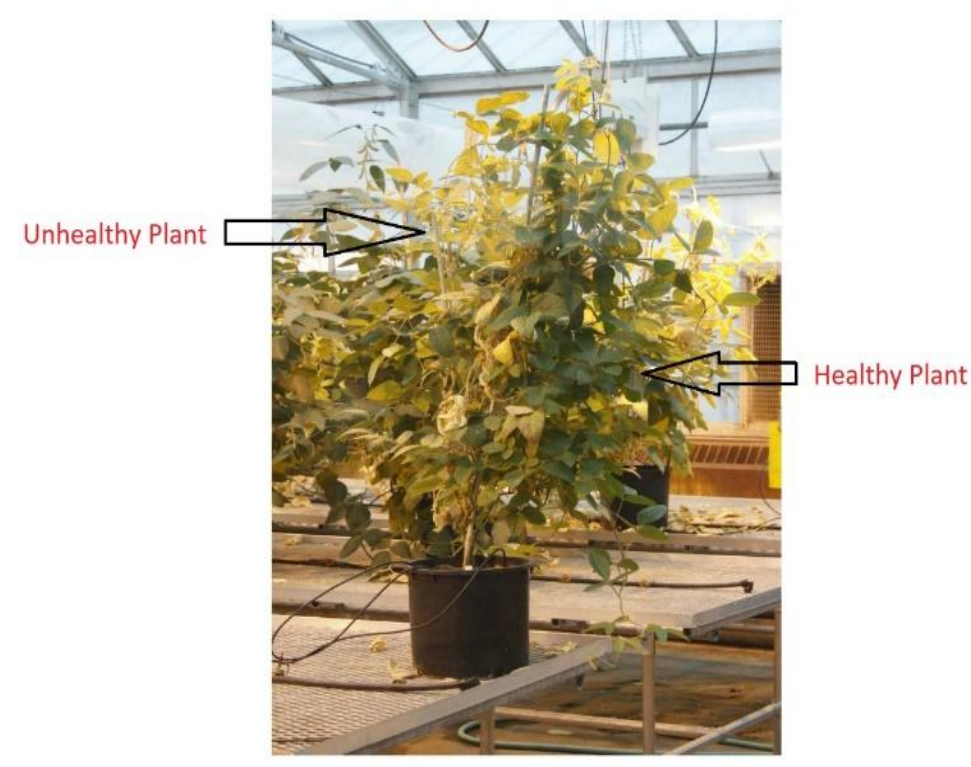

Figure 10. Normal Color Picture 


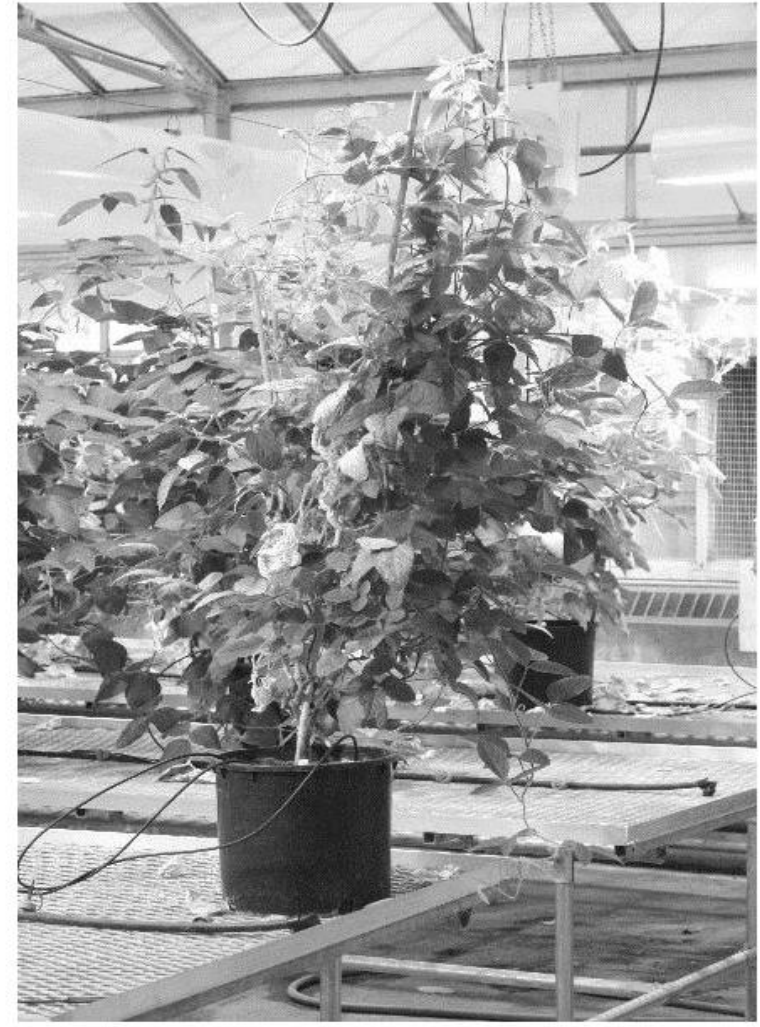

Red Wavelength Image

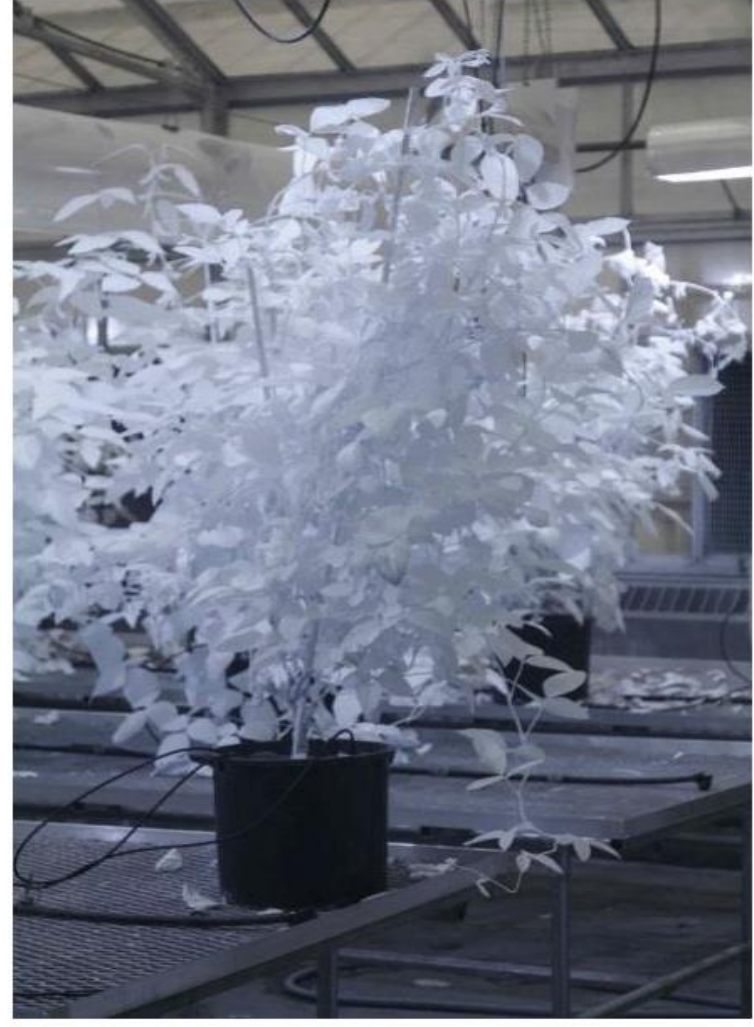

Near-Infrared Image

Figure 11. Two types of Images taken by choosing a certain color

NDVI can be created using equation 1 as follows:

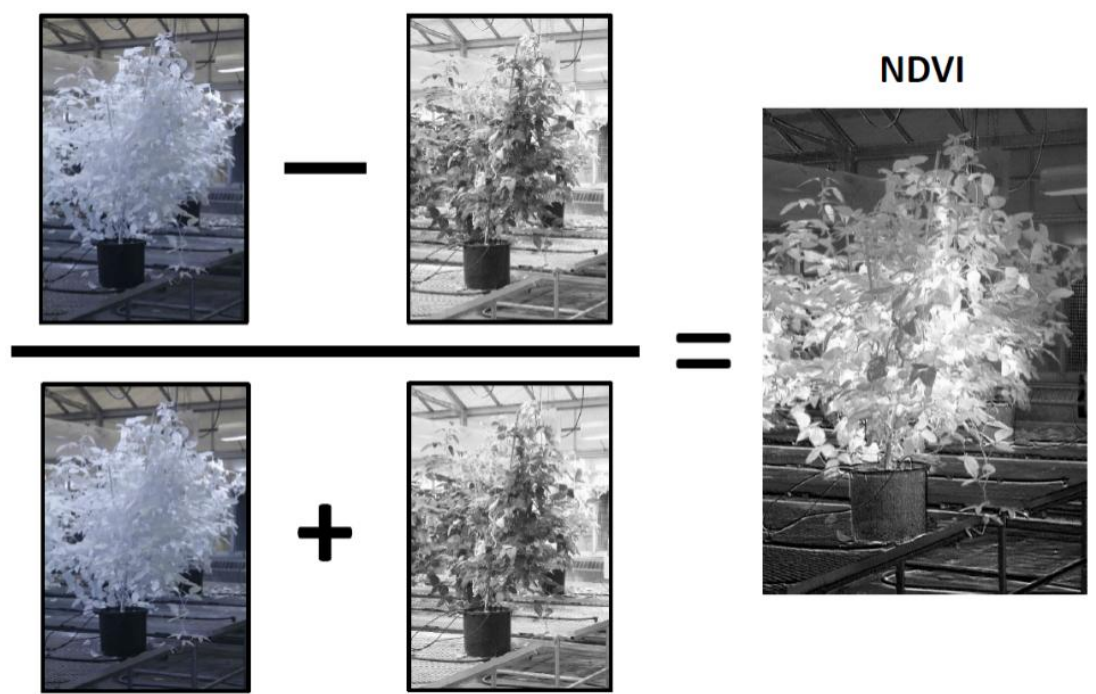

Figure 12. A general formula to calculate NDVI 
Vol. 7, Issue 11, November 2018

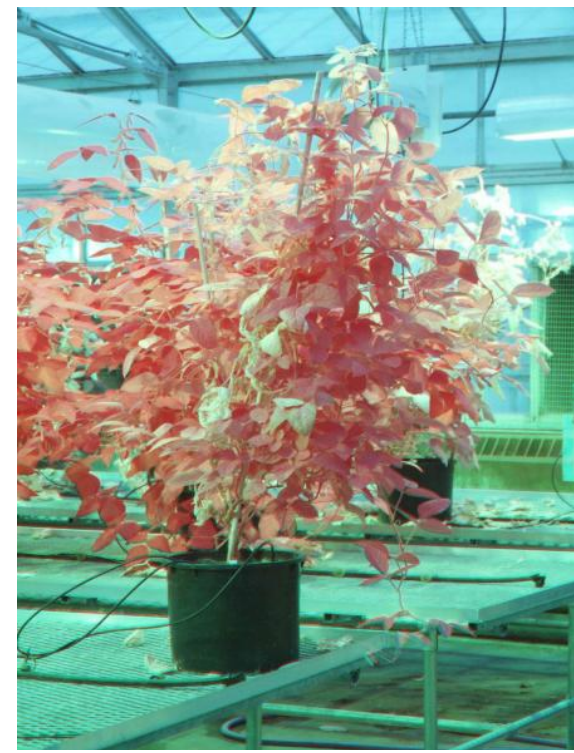

Figure 13. NIR, red and green wavelength image color composite

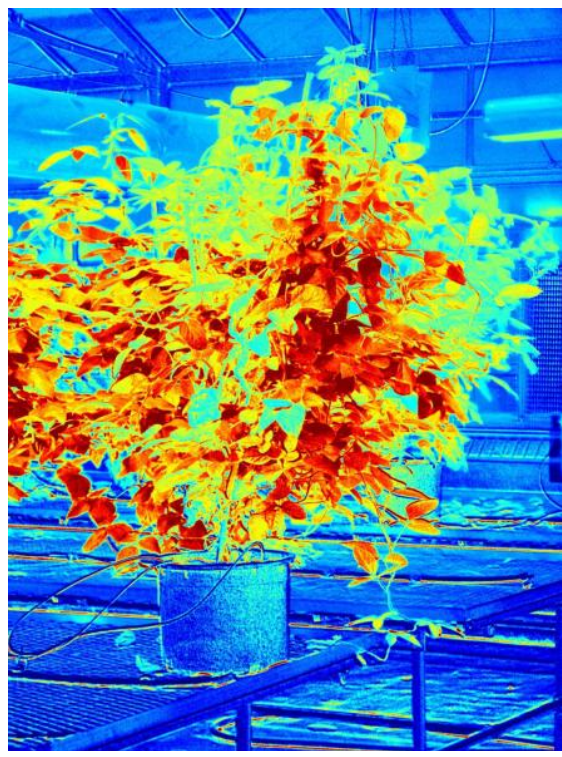

Figure 14. NDVI with red $=$ High values (Healthy) with blue $=$ Low values

\subsection{SPRINKLING SYSTEM}

Generally, the sprinkling system is attached to the lower region of the UAV which as a nozzle beneath the pesticide tank to sprinkle the pesticide towards downstream. The sprinkling system as two modules one is sprinkling system itself and second one is Controller. The sprinkling system contains the spraying content (pesticides or fertilizers) and a nozzle for spraying. Second one is controller used to activate the nozzle of the sprayer. A pressure pump is a component of the sprinkler system which pressurizes the pesticide to flow through the nozzle. A motor driver integrated circuit is used to pressure the pump as per the requirement. The same system can be used for irrigation by switching out the pesticide tank with a water tank.

\section{Conclusion ANd Future Scope}

UAV based crop monitoring system which surveys the crops and detects pests or crop defects using IR imagery and image processing algorithms will be implemented in a cost-effective manner with a robust and efficient design. An efficient and affordable UAV based crop monitoring and protection system will be made accessible to farmers in order to improve their yield. If it can be subsidized, then distribution throughout the country will be made possible and the state of Indian agriculture will be revolutionized and there will be a drastic improvement.

\section{REFERENCES}

[1] Chappelle, E., Kim, M. and McMurtrey, J. (1992) Ratio Analysis of Reflectance Spectra (RARS): An Algorithm for the Remote Estimation of the Concentrations of Chlorophyll A, Chlorophyll B, and Carotenoids in Soybean Leaves.Remote Sensing of Environment, 39, $239-247$. http://dx.doi.org/10.1016/0034-4257(92)90089-3

[2] Pinter, P., Hatfield, J., Schepers, J., Barnes, E., Moran, M.S., Daughtry, C., et al. (2003) Remote Sensing for Crop Management. Photogrammetric Engineering and Remote Sensing, 69, 647-664 http://dx.doi.org/10.14358/PERS.69.6.647

[3] Slaton, M., Hunt, E. and Smith, W. (2001) Estimating near Infrared Leaf Reflectance from Leaf Structural Characteristics. American Journal of Botany, 88, 278-284. http://dx.doi.org/10.2307/2657019

[4] Olivier, M., Heming, S. and Adams, G.G. (2003) Exploring the Spatial Variation of Take-All (Gaeumannemyces graminis var. tritici) for SiteSpecific Management. In: 4th European Conference on Precision Agriculture Wageningen Academic Publishers, Berlin, 481-486.

[5] Moshou, D., Bravo, C., West, J., Wahlen, S., McCann, A. and Ramon, H. (2004) Automatic Detection of Yellow Rust in Wheat Using Reflectance Measurements and Neural Networks. Computers and Electronics in Agriculture, 44, 173-188. http://dx.doi.org/10.1016/j.compag.2004.04.003

[6] Oerke, E.-C., Steiner, U., Dehne, H.-W. and Lindenthal, M. (2006) Thermal Imaging of Cucumber Leaves Affected by Downy Mildew and Environmental Conditions. Experimental Botany, 57, 2121-2132. http://dx.doi.org/10.1093/jxb/erj170

[7] C. Zhang, J. M. Kovacs, "The application of small unmanned aerial systems for precision agriculture: a review", Precision Agriculture, Springer, 2012.

[8] MIT Technology Review, "Agricultural Drones. Relatively cheap drones with advanced sensors and imaging capabilities are giving farmers new ways to increase yields and reduce crop damage", http://www.technologyreview.com/featuredstory/526491/agricultural-drones/, 2015.

[9] I. Colomina, P. Molina, "Unmanned aerial systems for photogrammetry and remote sensing: a review", ISPRS Journal of Photogrammetry and Remote Sensing, June 2014. 
Vol. 7, Issue 11, November 2018

[10] D. Anthony, S. Elbaum, A. Lorenz, C. Detweiler, "On Crop Height Estimation with UAVs", 2014 IEEE/RSJ International Conference on Intelligent Robots and Systems.

[11] J. Primicerio, S. F. Di Gennaro, E. Fiorillo, L. Genesio, E. Lugato, A. Matese, F. P. Vaccari, “A flexible unmanned aerial vehicle for precision agriculture", Precision Agriculture, Springer, 2012

[12] L. Hassan-Esfahani, A. Torres-Rua, A. M. Ticlavilca, A. Jensen, M. McKee, "Topsoil Moisture Estimation for Precision Agriculture Using Unmanned Aerial Vehicle Multispectral Imagery”, 2014 IEEE International Geoscience and Remote Sensing Symposium, 2014. 